\section{OP0276 DENDRITIC CELL-DERIVED IL-27 REGULATES THE MAGNITUDE OF INDUCIBLE ECTOPIC GERMINAL CENTRES BUT FAILS TO DOWNMODULATE IL-17 PRODUCTION IN CD4 T CELLS FROM PATIENTS WITH SJÖGREN'S SYNDROME}

Davide Lucchesi ${ }^{1}$, Rachel Coleby ${ }^{1}$, Elena Pontarini ${ }^{1}$, Edoardo Prediletto ${ }^{1}$, David Hill $^{2}$, Alicia Derrac Soria ${ }^{2}$, Felice Rivellese ${ }^{1}$, Costantino Pitzalis ${ }^{1}$, Simon Jones ${ }^{2}$, Gareth Jones ${ }^{3}$, Michele Bombardieri ${ }^{1} .{ }^{1}$ William Harvery Research Institute, Queen Mary University of London, Experimental Medicine and Rheumatology, London, United Kingdom; ${ }^{2}$ Cardiff University, School of Medicine, Cardiff, United Kingdom; ${ }^{3}$ University of Bristol, School of Cellular and Molecular Medicine, Bristol, United Kingdom

Background: Approximately 30\% of Sjögren's Syndrome (SS) patients develop Ectopic Lymphoid Structures (ELS) in their salivary glands (SG). ELS play an active role in autoimmunity and contribute to the development of MALT lymphoma. Interleukin 27 (IL-27) exerts key immunomodulatory actions on CD4 T cells with both pro and anti-inflammatory roles but its role in the formation and regulation of ELS in the salivary glands of SS is unknown.

Objectives: We first used a murine model of inducible SG ELS to elucidate the role of IL-27 and its interaction with IL-17 in the regulation of ELS formation and function. We then extended our observations on a cohort of SS patients to identify IL-27 cellular source, target cells and functional properties in modulating peripheral and lesional CD4 T cells function.

Methods: To trigger ELS formation a single dose of reporter-encoding adenovirus was delivered directly to the SG of wild-type (WT) and IL-27RA-deficient (KO) mice. For IL-17 blockade anti-mouse IL-17A antibody was administered systemically. ELS development and peripheral immune responses were tracked by immuno-histopathology, FACS, and GPCR. Minor SG biopsies were collected from SS and non-specific sialadenitis (sicca) patients. Peripheral blood mononuclear cells (PBMC) isolated from patients and age/sex matched healthy donors (HD). For in vitro experiments PBMCs, isolated CD4 T cells and parotid gland MCs were incubated with IL-27 and analysed by FACS for CD4 T cell subsets while cytokines levels were measured intracellularly by FACS and in culture supernatants. Tissue IL-27 was assessed in SS SG sections by multicolour immunofluorescence to identify IL-27 producing cells.

Results: In WT mice, SG ELS formation was preceded by an upregulation of IL27p28 and infiltration of IL-27 producing cells (CD11b+ first followed by CD4 and CD8 $T$ cells). KO mice displayed larger, more abundant ELS in the SG with more germinal centres and higher levels of ELS-related genes (Cxc/13, Cc/19, Ltb, Aid) compared to WT mice. During ELS formation, KO mice had an uncontrolled SG Th17 response and systemic IL-17A blockade caused reduction in ELS size and in the expression of ELS-related genes. In SS patients SG and serum, we observed higher expression levels of IL-27 transcripts and protein, respectively, compared to sicca, while SG IL-27 was selectively increased in the ELS+ subset of SS. Immunofluorescence staining for IL-27 revealed its presence primarily in the T cell rich areas of SG ELS with frequent co-localization with DC-LAMP+ dendritic cells. Finally, while IL-27 was able to significantly downregulate IL-17 production in HD, CD4 T cells from patients with SS failed to downregulate IL-17 but showed an aberrant IFN $\gamma$ release upon IL-27 incubation. We did not observe any difference in IL-27R expression or downstream STAT1/3 phosphorylation between SS and HD.

Conclusion: IL-27 is a critical regulator of the magnitude of the germinal centre response in the SG by restricting Th17 expansion. Both in murine inducible ELS and in patients with SS, dendritic cells appear as the main cellular source of IL-27. Although SG IL-27 was increased in the ELS+ subset of SS, it consistently failed to downregulate IL-17 release in CD4 T cells from SS patients suggesting that a profound dysregulation of the IL-27/L-17 axis play an important role in ELS formation in this condition.

Disclosure of Interests: Davide Lucchesi: None declared, Rachel Coleby: None declared, Elena Pontarini: None declared, Edoardo Prediletto: None declared, David Hill: None declared, Alicia Derrac Soria: None declared, Felice Rivellese: None declared, Costantino Pitzalis Grant/research support from: Celgene, Simon Jones: None declared, Gareth Jones: None declared, Michele Bombardieri Grant/research support from: Celgene, Consultant for: Medimmune DOI: 10.1136/annrheumdis-2019-eular.5889

\section{OP0277 \\ RNA SEQUENCING AND MACHINE LEARNING TECHNIQUES PREDICT MAJOR ORGAN INVOLVEMENT IN PATIENTS WITH SYSTEMIC LUPUS ERYTHEMATOSUS}

Anastasia Filia ${ }^{1}$, George Bertsias ${ }^{2}$, Nikolaos Panousis ${ }^{3}$, Dionisis Nikolopoulos ${ }^{1}$, Emmanouil Dermitzakis ${ }^{3}$, Dimitrios Boumpas ${ }^{1,4}$. ${ }^{1}$ Biomedical Research Foundation Academy of Athens, Athens, Greece; ${ }^{2}$ University of Crete, Medical School, Heraklion, Greece, ${ }^{3}$ University of Geneva, Medical School, Geneva, Switzerland; ${ }^{4}$ National and Kapodistrian University of Athens, Athens, Greece

Background: Both clinically and molecularly, Systemic Lupus Erythematosus (SLE) is a heterogeneous disease with non-synchronous multi-organ involvement of varying severity, and alternating periods of remission and flares. There is an unmet need for a blood-based- "liquid biopsy" to predict prognosis of the disease. Objectives: To detect the smallest set of genes predicting SLE organ involvement and disease activity using RNA-sequencing data derived from whole blood cells from 150 SLE patients and machine learning techniques.

Methods: Disease activity was measured by the SLE disease activity index-2000 (SLEDAI-2K) and by organ involvement (treated as binary outcomes). SLEDAI2K: mild-moderate disease (SLEDAI 0-8), severe (SLEDAI $\geq 8$ ). Organ involvement: major (renal, heart, lung, central nervous system \& SLEDAI-2K $>6$ ) and minor (all others). The RNA-sequencing dataset was pre-processed to assemble 20,368 genes and then split in training/validation data. Two feature selection steps (edge $R$ and recursive feature elimination) were used to remove noise and keep the smallest set of genes which best predicts each outcome. Different prediction models were fit to identify which one performs best using the gene signature selected in the previous step.

Results: Two gene signatures were kept after feature selection to predict each of the two outcomes (25 genes for organ involvement; 50 genes for SLEDAI-2K). Organ involvement was predicted with high accuracy (accuracy $=0.89$, sensitivity $=0.89$, specificity $=0.88$ in the validation data) using the elastic net generalised linear model. Among the 25 best predictors were MPO, ITGA3 and CD38. SLEDAl-2K could not be predicted with high accuracy (accuracy 0.75 , sensitiv ity $=0.79$, specificity $=0.67$ ) using the neural network model. Performance was still the same even when 1648 genes (after first feature selection step) were used as predictors of SLEDAI-2K. The performance of these models will also be tested in an independent test dataset once available (currently undergoing sequencing). Conclusion: The model predicting organ involvement performed better compared to the model predicting SLEDAl-2K. This could be attributed to the fact that certain disease manifestations are not currently included in SLEDAI-2K. Furthe analysis and functional laboratory experiments of those genes will help to identify biomarkers for more accurate assessment of disease activity and prognosis in the clinic.

Acknowledgement: This work was supported by FOREUM, SYSCID and ERCAdvanced Grant.

Disclosure of Interests: None declared

DOI: 10.1136/annrheumdis-2019-eular.4558

\section{OP0278 BIOMARKER PROFILING REVEALS NOVEL MECHANISTIC INSIGHTS INTO USTEKINUMAB THERAPEUTIC RESPONSES IN SYSTEMIC LUPUS ERYTHEMATOSUS}

Matteo Cesaroni ${ }^{1}$, Loqmane Seridi ${ }^{1}$, Jarrat Jordan ${ }^{1}$, Kristen Sweet ${ }^{1}$, MA Keying ${ }^{1}$ Carol Franks ${ }^{1}$, Jessica Schreiter ${ }^{1}$, Peter Lipsky ${ }^{2}$, Ronald van Vollenhoven ${ }^{3}$, Bevra H. Hahn ${ }^{4}$, Shawn Rose ${ }^{1}$, Frederic Baribaud ${ }^{1}$, Matthew J Loza ${ }^{1}$, Kim Campbell ${ }^{1}$, George Tsokos ${ }^{5} .{ }^{1}$ Janssen Research and Development, LLC, Spring House, United States of America; ${ }^{2}$ AMPEL BioSolutions, LLC, Charlottesville, United States of America; ${ }^{3}$ Amsterdam Rheumatology and Immunology Center, Amsterdam, Netherlands; ${ }^{4}$ University of California, Los Angeles, Los Angeles, United States of America; ${ }^{5}$ Beth Isreal Deaconess Medical Center, Harvard Medical School, Boston, United States of America

Background: Systemic lupus erythematosus (SLE) is a heterogenous autoimmune disease that causes progressive organ damage. The cytokines type I interferon (IFN-I), IL-12 and IL-23 have all been shown to contribute to SLE pathogenesis. We previously reported that treatment with ustekinumab (UST), an anti-IL-12/23 p40 neutralizing monoclonal antibody, improved global and organspecific measures of disease activity in a randomized, placebo (PBO)-controlled study of patients with active SLE (NCT02349061). ${ }^{1}$ Here, we utilized biomarke data from this clinical study to elucidate the mechanism of action of UST in SLE. Objectives: We aimed to determine whether modulation of IL-12, IL-23, or both cytokines was associated with clinical efficacy, and to ascertain whether UST treatment could modulate IFN-I or improve disease activity in patients exhibiting an elevated IFN-I signature at baseline.

Methods: A Phase 2, placebo (PBO)-controlled study enrolled 102 patients with seropositive SLE and active disease despite standard-of-care therapy. ${ }^{1} P$ atients were randomized 3:2 to receive UST IV $\sim 6 \mathrm{mg} / \mathrm{kg}$ or PBO at week 0 , then subcutaneous injections of $90 \mathrm{mg}$ UST q8w or PBO. Whole blood RNA from PAXgene tubes and serum were collected over 24 weeks. Age and sex-matched healthy controls were also studied. Serum IFN- $\gamma$, and IL-17A, IL-17F and IL-22 levels were quantified by ELISA as indicative of the IL-12 and IL-23 pathways, respectively, and an IFN- $\alpha$ ELISA was utilized to quantify the IFN-I pathway. Whole blood RNA was assessed for gene expression by microarray. Two Th1 $17^{2,3}$, an IFN- $\gamma^{4}$ gene signature and 21gene IFN-I signature (IGS) ${ }^{5}$ were analyzed. SLE Responder Index (SRI)-4 at week 24 was used to define UST response (UST-R) and non-response (UST-NR).

Results: Serum IL-17A, IL-17F and IL-22 levels and Th17 gene signature levels in blood remained largely stable over the course of 24 weeks in all treatment groups. In contrast, UST-R was associated with a durable reduction in IFN- $\gamma$ protein and IFN- $\gamma$ gene signature levels relative to baseline, which was not observed 